\title{
Aerodynamics of a cycling wheel in crosswind by coaxial volumetric velocimetry
}

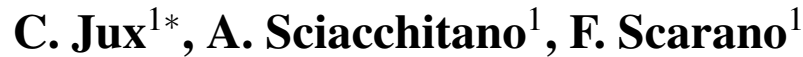 \\ ${ }^{1}$ Delft University of Technology, Dept. of Aerospace Engineering - Aerodynamics, Delft, The Netherlands \\ *C.Jux@tudelft.nl
}

\begin{abstract}
The aerodynamic characteristics of a modern road cycling wheel in crosswind are studied through force measurements and 3D velocimetry in TU Delft's Open Jet Facility. The performance of the $62 \mathrm{~mm}$ deep rim is evaluated for two tire profiles, and yaw angles up to $20^{\circ}$. All measurements are executed at 12.5 $\mathrm{m} / \mathrm{s}(45 \mathrm{~km} / \mathrm{h})$ freestream- and wheel-rotational velocity. The wheel's rim-tire section in crosswind is found to behave similar to an airfoil at incidence, ultimately resulting in a reduction of the wheel's aerodynamic resistance with increasing yaw angle magnitude. This trend, also referred to as the sail-effect, is limited by the stall angle of the tire-rim profile. The stall angle is found to be dependent on the tire surface texture and varies between $14^{\circ}$ and $20^{\circ}$.
\end{abstract}

\section{Introduction}

Aerodynamic drag is the major resistance a cyclist needs to overcome at speeds of $40 \mathrm{~km} / \mathrm{h}$ and beyond. In fact, the aerodynamic drag accounts for approximately $90 \%$ of a rider's total resistance in this speed regime which is typical for road racing (Kyle and Burke, 1984). The wheels contribute to about $10 \%$ of the total aerodynamic drag (Greenwell et al,, 1995), making them a crucial element in any cycling-performance optimization.

Focusing on road cycling, a rider is exposed to a variety of wind conditions, affecting the perceived yaw angle. The concept of wind-averaged drag (WAD, Cooper, 2003) accounts for the statistically anticipated yaw-angle distribution experienced by a ground vehicle. The higher the vehicle velocity, the smaller the maximum yaw angle perceived for a given wind speed. For professional road cycling speeds, the probability of exceeding $20^{\circ}$ yaw angle is reported to be less than 10\% (Brownlie et al, 2010; Barry, 2018). Vice versa, a rider spends $90 \%$ of the time at yaw angles below $20^{\circ}$.

Aerodynamically designed wheels used in road racing (thus, excluding time-trial and triathlon specific multi-spoke and disc wheels) are characterized by a $40-80 \mathrm{~mm}$ deep rim section, that connects via a multitude of thin spokes to a cylindrical hub. In comparison to classical, shallow rim profiles, the deep-section rim fulfills two functions: on one hand, it streamlines the tire shape, effectively reducing the tire-rim drag coefficient. On the other hand, it generates - similar to a sail - a side force perpendicular to the relative wind direction. A component of this force points in the riding direction, effectively "pulling" the wheel forwards and thereby further reducing its drag coefficient for non-zero yaw angles. The latter effect is known as the "sail-effect" in literature, and it can result in a negative net drag force for wheels at high yaw angles (Lukes et al., 2005; Barry et al., 2012; Malizia and Blocken, 2020)a).

Designing a wheel for optimal performance is, however, not solely about minimizing (wind-averaged) drag. As Barry et al. (2012) point out, large side forces on wheels yield strong steering moments and can be a concern of maneuverability. Similar concerns can be expressed when discussing the stall behavior for wheels at large yaw angles.

In addition to the rim-geometry, the aerodynamic characteristics of a wheel depend on the interaction of rim and tire. Crane and Morton (2018) investigate this interaction with specific attention to tire width relative to the rim. Besides tire width, data from wheel manufacturers suggests that the tire profile is equally decisive for the rim-tire interaction, because the tire's surface texture conditions the boundary layer development for the flow over the wheel (Cant, 2014). Complementing the analysis of Crane and Morton (2018), this work includes the comparison of a slick tire to a slightly profiled tire for a fixed tire width. 
In the discussion of cycling wheel aerodynamics it is common practice to study wheels in isolation. Experimental studies in the field focus predominantly on balance measurements (e.g. Zdravkovich, 1992; Greenwell et al,, 1995; (Tew and Sayers, 1999), whereas flow topology data remains undocumented to the best of the authors' knowledge. Such data is readily available from computational fluid dynamics (CFD) analysis, which presents an active line of research in the context of cycling wheel aerodynamics (Godo et al, 2010); Malizia et al, 2019; Malizia and Blocken, 2020b). Authors developing numerical tools for the aerodynamic analysis of cycling wheels, however, have to rely on the experimentally documented force data for validation of their simulations. This practice becomes problematic when reported drag measurements for the same wheel show variations of up to $300 \%$, as observed by Godo et al. (2010). The large force sensitivity is ascribed to variations in the experimental setup, including tire choice, free-stream turbulence, wheel support design and wind tunnel blockage.

In view of the gaps and discrepancies in literature, the present work provides an experimental analysis of the flow topology on a state-of-the-art bicycle wheel in crosswind by means of coaxial volumetric velocimetry (CVV, Schneiders et al, 2018). The change in flow topology with yaw angle and tire selection is documented. Additionally, the hypothesized "sail-effect" theory is scrutinized by analysis of the pressure distribution near the rim-tire surface.

\section{Definitions \& terminology}

Prior to presentation of the experimental apparatus and procedures in the subsequent section, we provide clear definitions of the relevant variables and coordinate systems. The provided definitions largely follow the work of Tew and Sayers (1999).

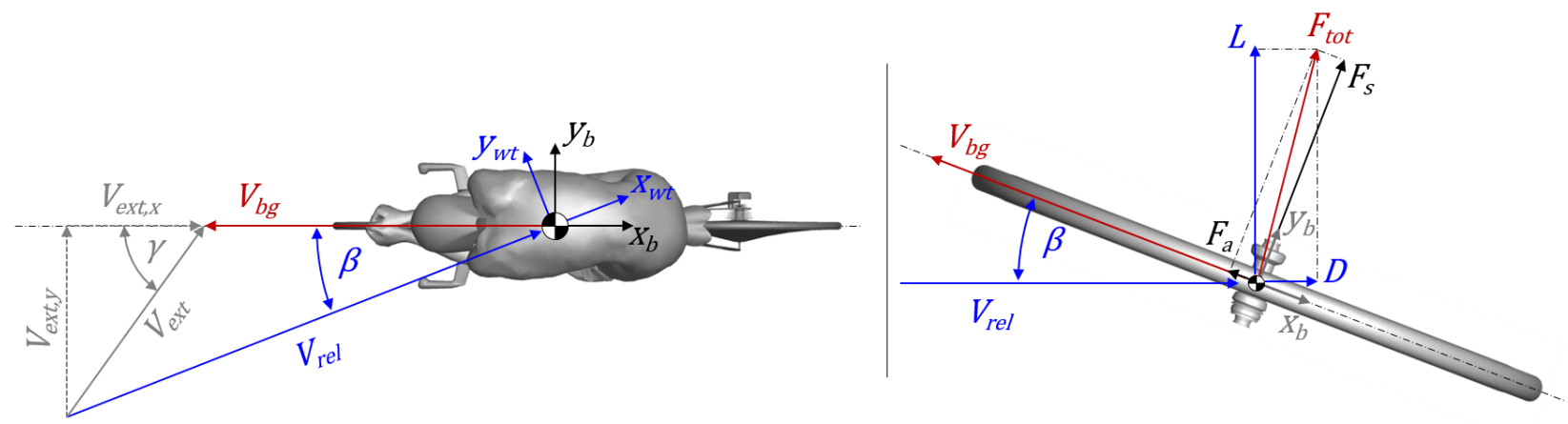

Figure 1: Definition of axes systems, flow angles and velocities for a cyclist moving at a velocity $V_{b g}$ relative to the ground (left). Relation of forces in wind and bike reference frames, illustrated on a bicycle wheel in top view (right).

Figure $\mathbf{W}$ (left) shows a cyclist moving at a velocity $V_{b g}$ relative to the ground. The direction of travel defines the main reference frame $\mathbf{X}_{b}$, pertaining to the bicycle, with the $x$-axis pointing opposite to the motion direction. In an external environment, the wind velocity $V_{e x t}$ and its direction $(\gamma$, the crosswind angle) relative to the cyclist's direction of motion are relevant for the definition of the aerodynamic problem. The vector difference of external wind $V_{e x t}$ and bicycle ground velocity $V_{b g}$ determines the relative wind velocity $V_{\text {rel }}$ experienced by the cyclist. The angle between the cyclist's motion axis and the relative wind velocity defines the yaw angle $\beta$. Let us define a second coordinate system $X_{w t}$ whose $x$-axis is aligned with $V_{r e l}$. The subscript ' $w t$ ' signals that this reference frame pertains to the wind-tunnel when testing in a laboratory environment, where the $x$-axis is always aligned with the relative wind direction.

Figure 1 (right) illustrates the resistive and lateral forces acting on a bicycle wheel. Classically, the drag force $D$ acts parallel to the free-stream direction defined by $V_{\text {rel }}$, whereas the lift force $L$ points perpendicular to it, combining to the resultant force $F_{t o t}$. More relevant for a cyclist, however, are the force components in the reference frame $\mathbf{X}_{b}$ connected to the direction of motion. The side force $F_{s}$ and the axial force $F_{a}$ are expressed as geometrical projections of $L$ and $D$ :

$$
\begin{aligned}
& F_{a}=D \cos (\beta)-L \sin (\beta) \\
& F_{s}=D \sin (\beta)+L \cos (\beta)
\end{aligned}
$$


It follows that a situation can arise in which $L \sin (\beta)>D \cos (\beta)$, resulting in a thrusting force which supports the cyclist's motion - the sail effect. In the remainder of this work, forces are always provided and discussed in the bicycle reference frame.

\section{Experimental apparatus and procedures}

Wind tunnel measurements are conducted at TU Delft's Open Jet Facility, an open jet, atmospheric wind tunnel with a $2.85 \times 2.85 \mathrm{~m}^{2}$ exit section. All measurements are executed at a freestream velocity of $12.5 \mathrm{~m} / \mathrm{s}$ $(45 \mathrm{~km} / \mathrm{h})$. The turbulence intensity in the test section, with a large-scale PIV seeding system installed in the settling chamber, is reported with $0.8 \%$ by Giaquinta (2018). An overview of the test setup is provided in Figure ฉ.
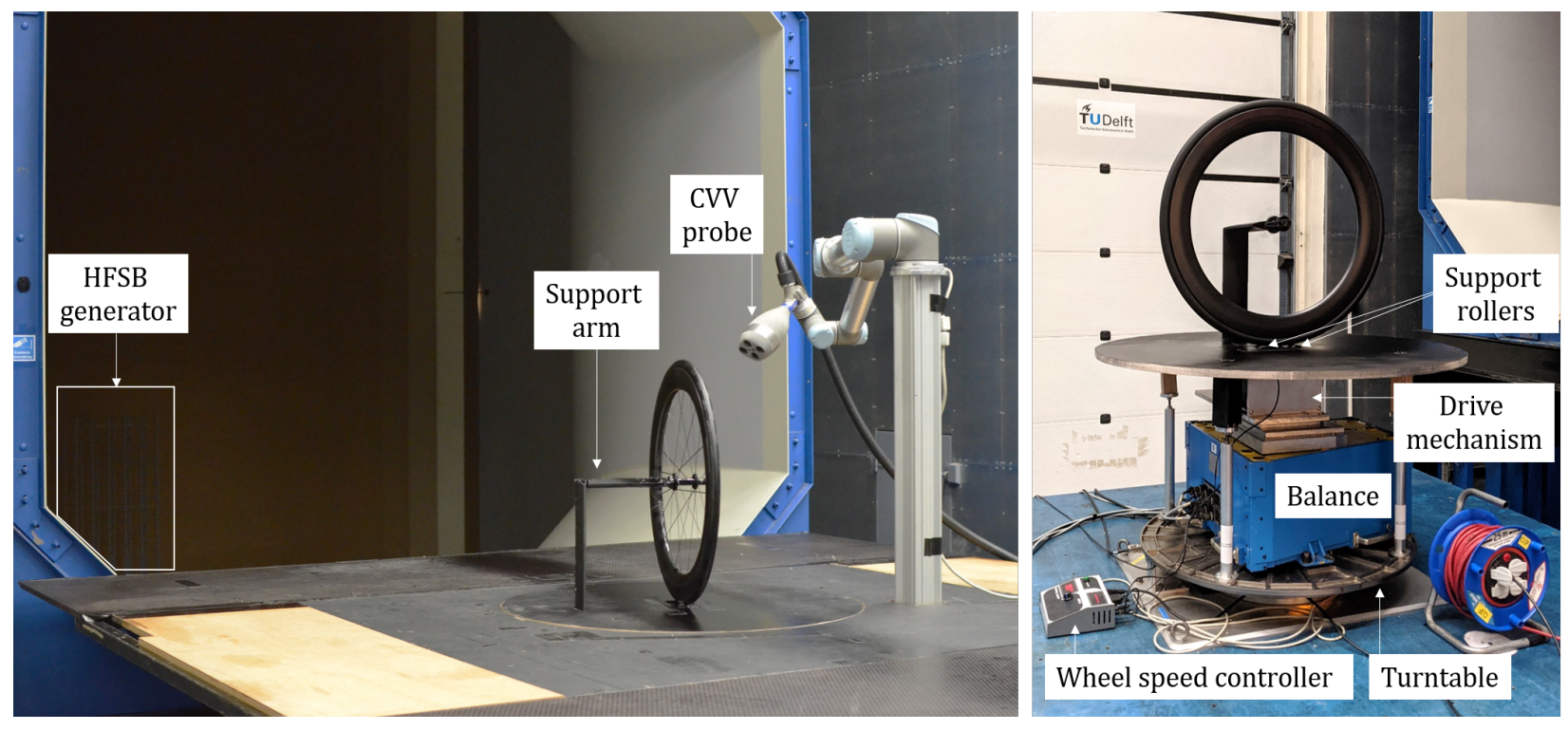

Figure 2: Experimental setup in the Open Jet Facility (left). Details of the mechanical systems installed in the wind tunnel, with surrounding floor plates removed (right).

\subsection{Wheel model \& mechanical setup}

A DT Swiss ARC 1100 Dicut DB 62 front wheel is installed in the test section. The 28 " $(700 \mathrm{~mm}$ diameter with tire fitted) wheel features a $62 \mathrm{~mm}$ deep and $27 \mathrm{~mm}$ wide carbon rim. While the wheel is designed to run with disc brakes, no brake discs were installed during the wind tunnel test. The clincher rim is fitted with a $25 \mathrm{~mm}$ tire, inflated at 7 bar. A Continental GP 5000 tire featuring a mild periodic surface pattern is chosen as a baseline tire, which is compared to a slick tire, a Continental GP TT. The difference in tire profile is shown in Figure 13. The grooved texture element on the baseline tire model is approximately 35 $\mathrm{mm}$ long, and $12 \mathrm{~mm}$ wide, with a $35 \mathrm{~mm}$ gap along the circumference between two subsequent elements.

The wheel is connected to the support structure using a standard $12 \times 100 \mathrm{~mm}$ through axle. The singlesided support holding the wheel from the left hand side is designed to enhance optical access for the velocimetry measurements.

The tire rests on two $70 \mathrm{~mm}$ diameter $(60 \mathrm{~mm}$ width) rollers embedded in the wooden ground plate. The rearward roller is driven by a variable speed DC motor to control wheel rotation. The wheel rotational speed is kept constant at $348 \mathrm{rpm}$, matching a ground velocity $\left(V_{b g}\right)$ of $12.5 \mathrm{~m} / \mathrm{s}$ equal to the freestream velocity. Wheel rotational speed and freestream velocity are both maintained constant at all times, and only the yaw angle is adjusted during the measurements.

The wheel branding is covered by black adhesive foil to limit background reflections in the PIV acquisition. A few circular ( $2 \mathrm{~mm}$ diameter) targets on the rim serve as reference markers to locate the model in the PIV data, as well as a means to verify the wheel rotational speed. 


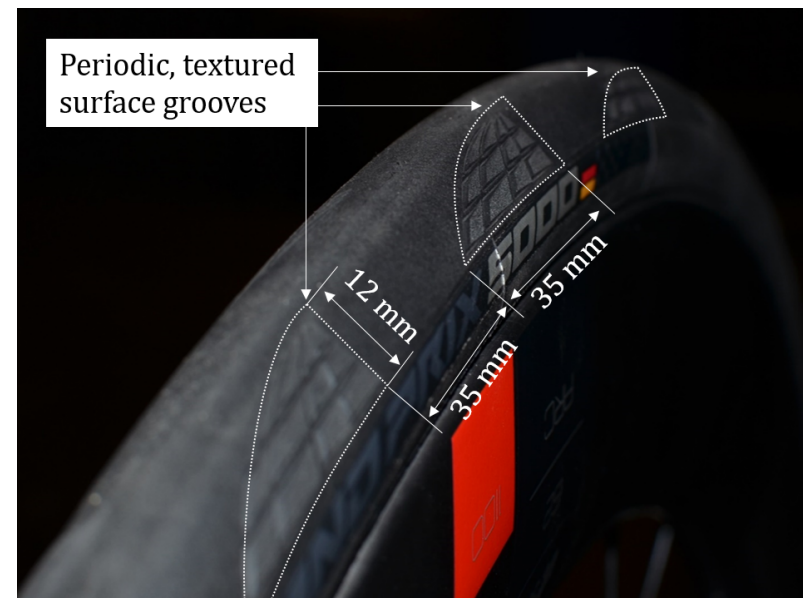

(a) Baseline tire, Continental GP 5000, with approximate indication of texture element size.

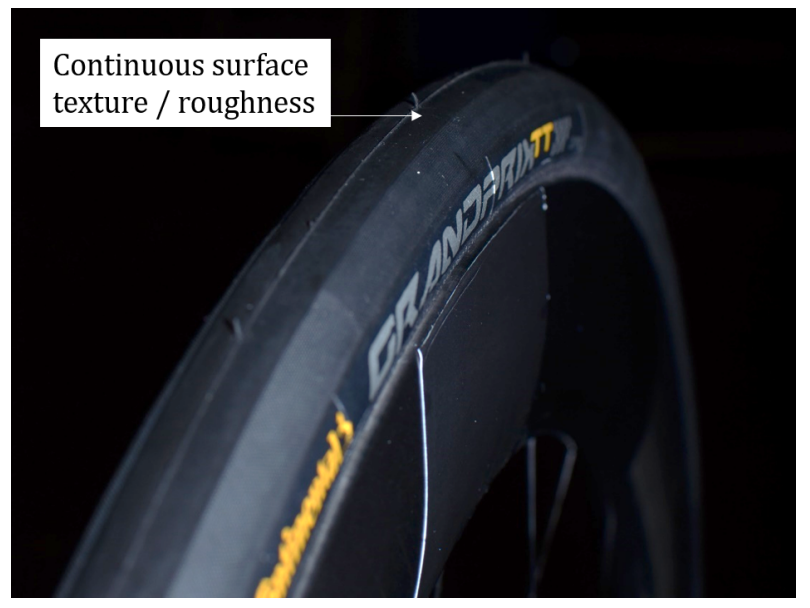

(b) Option tire, Continental GP TT

Figure 3: Fotographs of the tested tires mounted on the wheel.

\subsection{Force measurement system}

The wheel model including the mechanical support and drive mechanism is installed on a 6-axis force balance situated underneath the ground plate. The balance acquires data at a frequency of $2 \mathrm{kHz}$, and its accuracy is reported with $0.06 \%(0.15 \mathrm{~N})$ of its full $250 \mathrm{~N}$ load capacity (Alons, 2008). Force data is acquired and averaged over $20 \mathrm{~s}$. The balance is placed on a turntable that controls the yaw angle. As such, the force measurements delivered by the balance system pertain to the bicycle wheel reference frame $\left(\mathbf{X}_{b}\right)$. The yaw angle is varied in a range of $\beta$ between $-22^{\circ}$ and $+22^{\circ}$ in steps of $2^{\circ}$. The arrangement of the components is illustrated in Figure $\square$ (right).

Prior to force measurements on the wheel, the forces on the isolated support are recorded for all yaw angles of interest. This data is subsequently subtracted from the measurements with the wheel installed to identify the forces generated by the wheel only.

\subsection{Velocimetry system}

Three-dimensional (3D) particle image velocimetry data is acquired by a coaxial volumetric velocimetry (CVV, Schneiders et al, 2018) system. A LaVision MiniShaker Aero CVV probe acquires image quadruples of $640 \times 476 \mathrm{px}^{2}$ at a frequency of $821 \mathrm{~Hz}$. 16,400 images are acquired for each yaw angle, equaling an acquisition period of $20 \mathrm{~s}$. The velocimeter is mounted on a Universal Robots UR5 robotic arm, allowing to keep a similar imaging position and distance relative to the wheel for all yaw angles.

Neutrally-buoyant helium filled soap bubbles (HFSB, Scarano et al, 2015) of $300-500 \mu \mathrm{m}$ diameter serve as tracer particles, which are provided by a $0.6 \times 0.8 \mathrm{~m}^{2}$ seeding rake installed in the wind tunnel settling chamber. The measurement domain spans a $30 \times 20 \times 20 \mathrm{~cm}^{3}$ volume near the most upstream section of the wheel. Data is only acquired on the leeward, respectively, the suction-side of the rim.

\subsubsection{Velocity measurement}

The wheel rotation results in unsteady reflections which are filtered from the particle images by means of a Butterworth frequency filter (Sciacchitano and Scarano, 2014), followed by a directional minimum filter on a local $5 \times 5 \mathrm{px}^{2}$ kernel. Pre-processed images are analyzed by the 3D Lagrangian particle tracking algorithm "Shake-The-Box" (STB, Schanz et al, 2016) as implemented in Davis 10.1. The scattered STB tracks are subsequently ensemble averaged by a linear fit of the velocity data in ellipsoidal cells of $6 \times 6 \times 30 \mathrm{~mm}^{3}$ $(x \times y \times z)$, analogous to the approach presented by Agüera et al. (2016). Anisotropic cells are selected to enhance spatial resolution in the horizontal directions, where velocity gradients are anticipated be stronger as compared to the vertical direction, for the section of the wheel imaged by the velocimetry system. The grid spacing is maintained constant at $1.5 \mathrm{~mm}$ in all axes. 


\subsubsection{Pressure evaluation}

Based on the time-averaged velocity data, the static pressure is evaluated following a dual-model approach previously presented by the authors (Jux et al, 2020). In the region of irrotational flow, Bernoulli's equation provides the local pressure and the necessary Dirichlet condition for the subsequent spatial integration of the pressure gradient in the rotational flow domain. Lastly, the static pressure in the flow is mapped onto rim and tire surface, using the local pressure gradient information.

\section{Results and discussion}

The analysis of the measurement data starts with the force measurements. Subsequently, the velocimetry data is analyzed, leading to the discussion of the pressure distribution.

\subsection{Force measurement}

Figure 4 compares the axial and lateral forces as function of yaw angle for the two tested tire options. The side force $F_{s}$ in Figure ta features a linear trend of decreasing force magnitude with increasing yaw for moderate angles within $|\beta| \leq 10^{\circ}$. In this range, the lateral force reduces by approx. $0.85 \mathrm{~N} /{ }^{\circ}$, independent of the selected tire. Interestingly, the point of zero side force is found at a small positive yaw angle of about $2^{\circ}$. For large yaw angle magnitudes beyond $10^{\circ}$, the slope tapers off with a clear difference between the tires: the (slick) option tire reaches higher side force magnitudes for both, large negative and positive yaw angles, whereas the side force for the (profiled) baseline tire tapers off at slightly smaller yaw angle magnitudes. This trend is well illustrated around $\beta=14^{\circ}$ where the force curves cross each other.

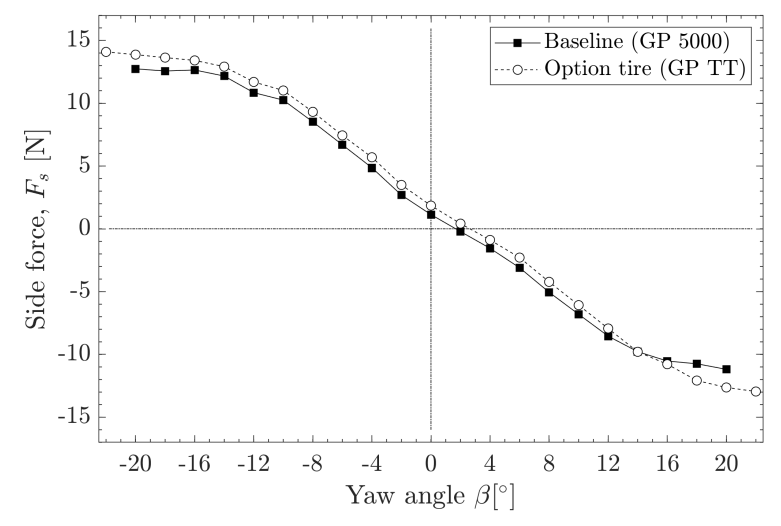

(a) Lateral force

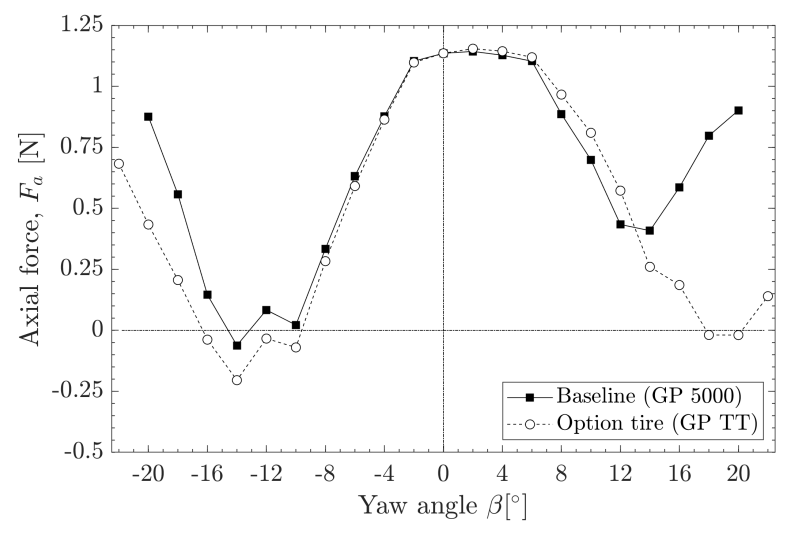

(b) Axial force

Figure 4: Force measurements on the isolated wheel versus yaw angle.

The axial force $F_{a}$ in Figure $4 b$ indicates a plateau of maximum resistance for small yaw angles ($2^{\circ} \leq \beta \leq 6^{\circ}$ ). The peak resistance of $1.12 \mathrm{~N}$ is found at $2^{\circ}$ yaw, corresponding with the location of zero side force in Figure 4a. Outside the indicated range, the axial force rapidly reduces for increasing yaw angle magnitudes. For negative yaw angles the tires behave similarly, with the axial force decreasing until $-10^{\circ}$ yaw, where a negative resistive force is measured, meaning the wheel is generating a propulsive force in this condition, albeit small in magnitude with about $0.1 \mathrm{~N}$. The trend of decreasing axial force is stagnating in the range of $-14^{\circ} \leq \beta \leq-10^{\circ}$, before the axial force rapidly increases again for even lower yaw angle values. This suggests that the flow over the wheel separates around $\beta=-12^{\circ}$. For yaw angles below $-10^{\circ}$ the (slick) option tire shows a lower axial force as compared to the baseline tire.

Differences between the two tire options are stronger for large positive yaw angles. The baseline tire initially shows a steeper reduction of the axial force with increasing yaw until $\beta=12^{\circ}$, where the trend turns, and the resistive force increases again for $\beta>14^{\circ}$. For positive yaw angles, the resistive force on the wheel with the baseline tire is always positive. In contrast, the axial force on the wheel with the option tire keeps reducing until $\beta=18^{\circ}$, although at a slightly slower rate. An increase in axial force is only observed for the highest tested yaw angle of $22^{\circ}$. Negative resistance is observed for $18^{\circ} \leq \beta \leq 20^{\circ}$. 
Despite the differences between the tire configurations, both cases show an asymmetric behavior against yaw angle: (1) the point of maximum axial force and minimum side force is found at $\beta=2^{\circ}$ rather than $0^{\circ}$. (2) Minima in axial force are different in magnitude and occur at different yaw angle magnitudes when comparing behavior in negative and positive yaw. Potential sources of this asymmetry are the non-symmetric wheel support, holding the wheel only from the left-hand-side (LHS); the blockage caused by the support structure of the PIV system on the wheel's right-hand-side (RHS); and the asymmetric design of the wheel's hub and spokes to accommodate a (centerlock) brake disc on its LHS.

The above analysis of the force data suggests nonetheless that the aerodynamic performance of the wheel is strongly dependent on the tire choice. The two tires behave similarly in the range of $-10^{\circ} \leq \beta \leq$ $10^{\circ}$, whereas for larger yaw angles the option tire features lower axial resistance, which even becomes negative, and thus acts as a (small) propulsive force. The lower resistive force of the option tire comes with an increase in lateral force, which is approximately $10 \%$ higher as compared to the wheel with the baseline tire for $|\beta|>14^{\circ}$. In the following we scrutinize the velocimetry data to link the observed differences to changes in the flow topology.

\subsection{Time-average velocity field}

For analysis of the flow topology, we extract slices of the 3D velocimetry data in a horizontal plane at hubheight $(z=342.5 \mathrm{~mm})$. The data shown in Figure $\square$ contains the velocity field upstream and past the leeward (suction) side of the rim. The velocity contours show the normalized axial velocity component in the bicycle wheel reference frame $\mathbf{X}_{b}$,

$$
u_{b}^{*}=\frac{u_{b}}{u_{b_{\infty}}}=\frac{u_{b}}{u_{w t_{\infty}} \cos (\beta)}
$$

where the subscript ' $\infty$ ' indicates free-stream conditions upstream of the wheel. The velocity contours are complemented by streamlines in the 2D planes. Additionally, for each yaw angle, the aerodynamic effect of the tire is illustrated by plotting the difference in the axial velocity component between the baseline and the option tire.

In straight ahead conditions ( $\beta=0^{\circ}$, Figure $5 \mathrm{a}$ ) the inflow decelerates towards the most upstream point of the tire. While one would expect the streamwise velocity component to stagnate at the tire surface, the finite velocity measurement reduces only to $u_{b}^{*} \approx 0.6$, which is attributed to spatial resolution effects. Alongside the rim-tire profile the flow accelerates reaching streamwise velocities in excess of $u_{b}^{*}=1.2$ at the thickest section of the rim. The wake of the rim is not well captured by the velocimetry data. The streamlines, however, suggest the presence of a separated wake zone just downstream of the rim. The differences between the tire options are very mild at $0^{\circ}$ yaw, in line with the balance data discussed previously.

The region of accelerated flow past the wheel gains in size and magnitude as the yaw angle increases. Consider e.g. Figure $5 \mathrm{~d}\left(\beta=8^{\circ}\right)$ where for both cases the axial velocity exceeds $130 \%$ of the inflow velocity as it passes the wheel. Comparing back to the straight ahead case in Figure 5a it is also noted that the location of maximum velocity moved upstream, approximately to the junction of rim and tire, whereas at $0^{\circ}$ yaw the maximum velocity is observed further downstream at the thickest point of the rim. Likewise, the zone of decelerated flow upstream of the wheel rotates towards the windward side as the yaw angle increases. The latter is seen well by following the unity contour of axial velocity $\left(u_{b}^{*}=1\right)$ which follows a counter-clockwise motion with increasing yaw angle.

The described trend continues for both tire cases until $\beta=12^{\circ}$ (Figure $5 \mathrm{~d}$ ). For greater yaw angles there is a substantial difference in the flow topology for the different tire options. Comparing the data in Figures $5 \mathrm{E}$ and 5H, the streamlines pertaining to the (slick) option tire in the right-hand-side plots suggest that the flow follows the largest part of the wheel contour. Instead, for the (textured) baseline tire in the left-hand-side plots, the streamlines only bend slightly around the tire but continue diverging from the rim surface as the flow passes the wheel. The different behavior is also visible in the delta plot in Figure 57, which indicates that the flow around the wheel with the option tire attains far greater axial velocity. While flow data closer to the rim surface is lacking for the baseline tire at these high yaw angles, the streamlines indicate that the flow separates from the tire, similar to a leading edge separation on aeronautical airfoil profiles at high angle of attack. Such leading edge separation results in a loss of lift (here, side force) and a simultaneous increase in drag (here, axial force) which is in line with the observations from the force data above. 

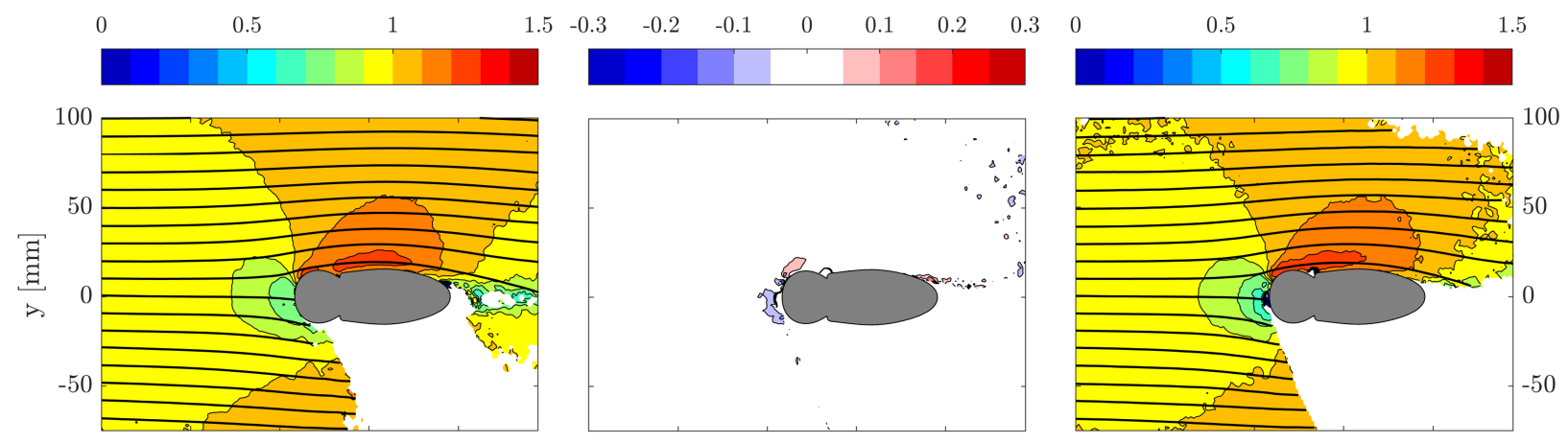

(a) $\beta=0^{\circ}$
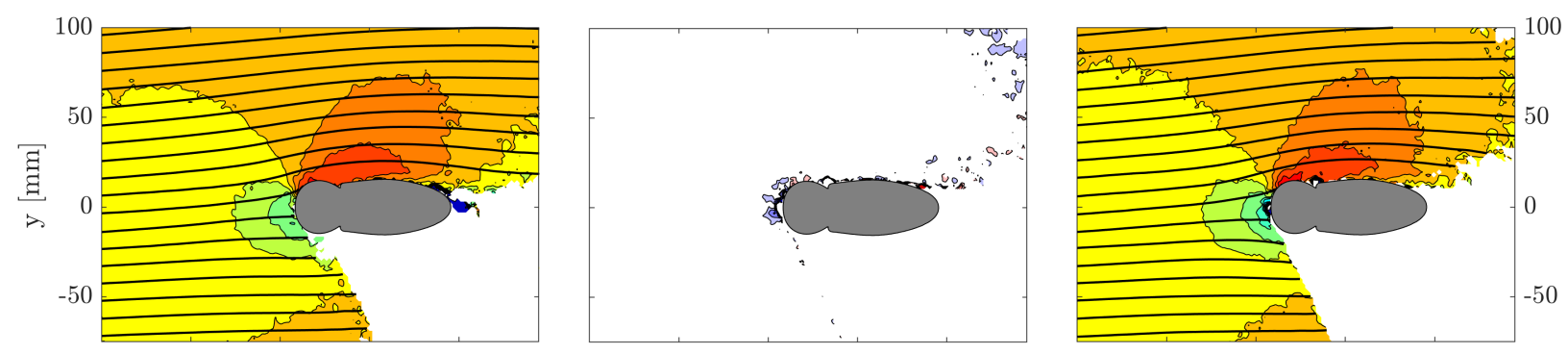

(b) $\beta=4^{\circ}$
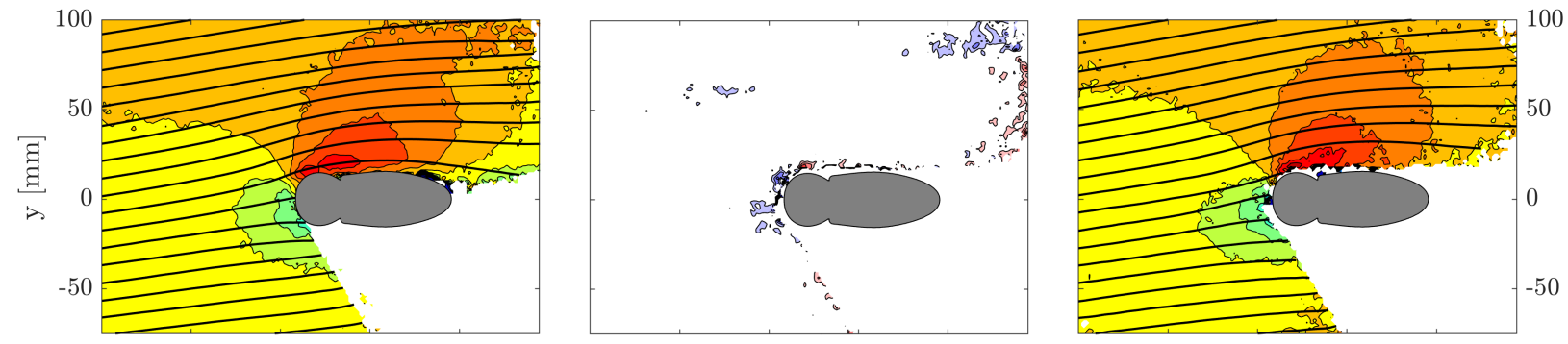

(c) $\beta=8^{\circ}$
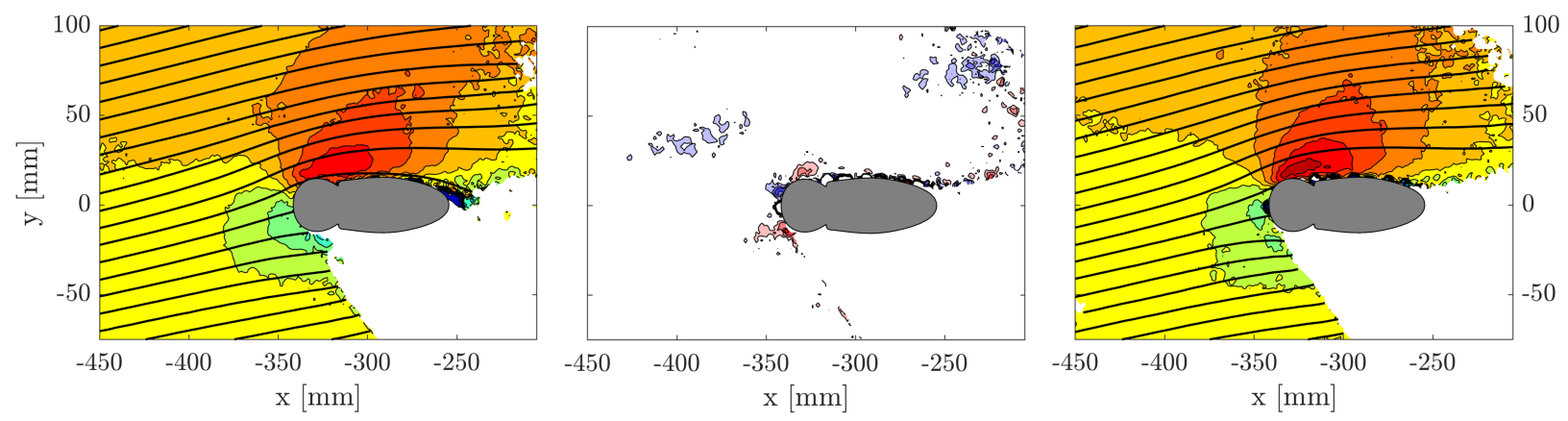

(d) $\beta=12^{\circ}$

Figure 5: Time-average velocity contours in $x y$-planes at hub height $(z=342.5 \mathrm{~mm})$, along with 2D surface streamlines. Velocity contours show the normalized axial velocity component in the bicycle wheel reference frame. (Left) Baseline tire. (Right) Option tire. (Center) Delta $=u_{\text {baseline }}-u_{\text {option. }}$. (Continues on next page) 

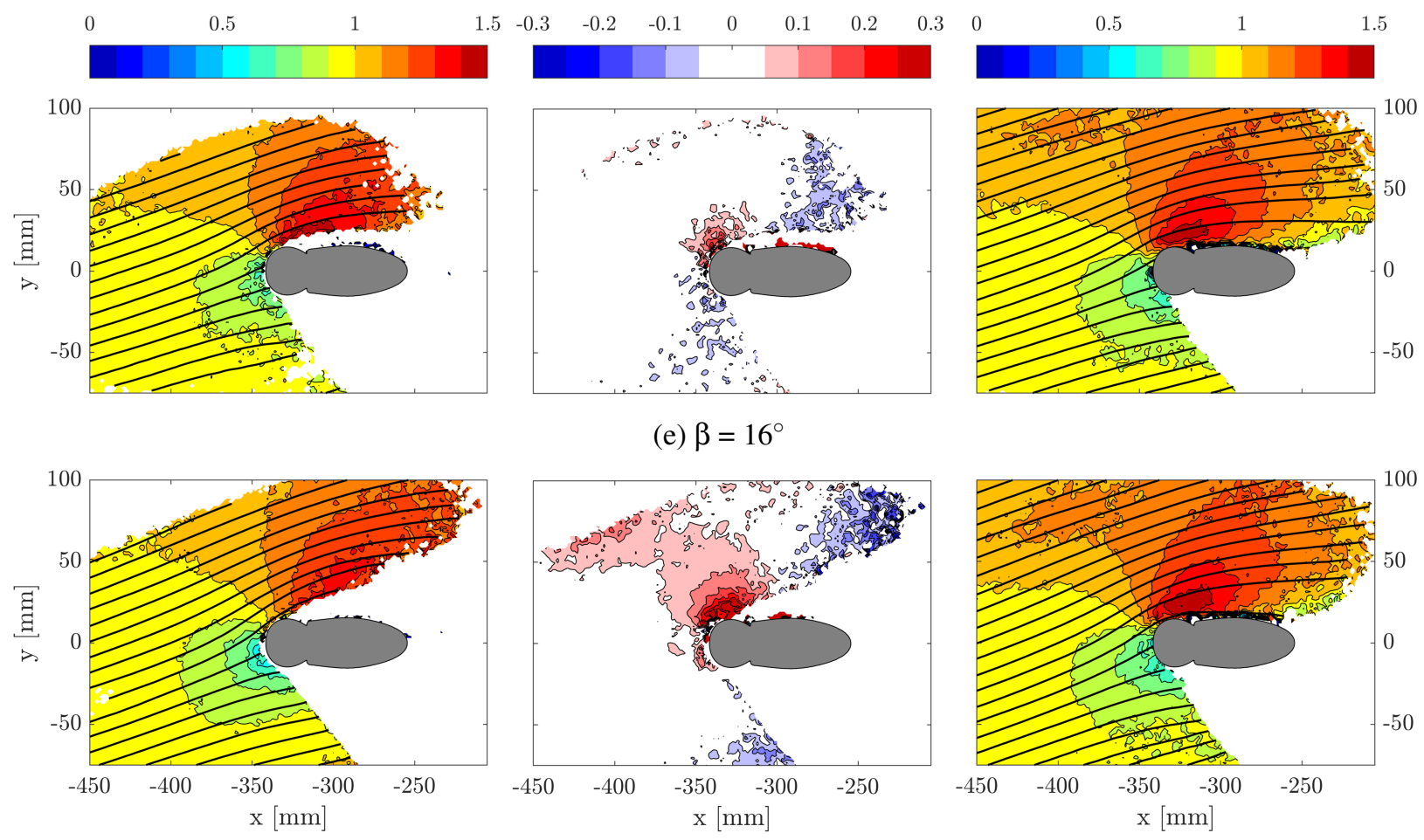

(e) $\beta=16^{\circ}$
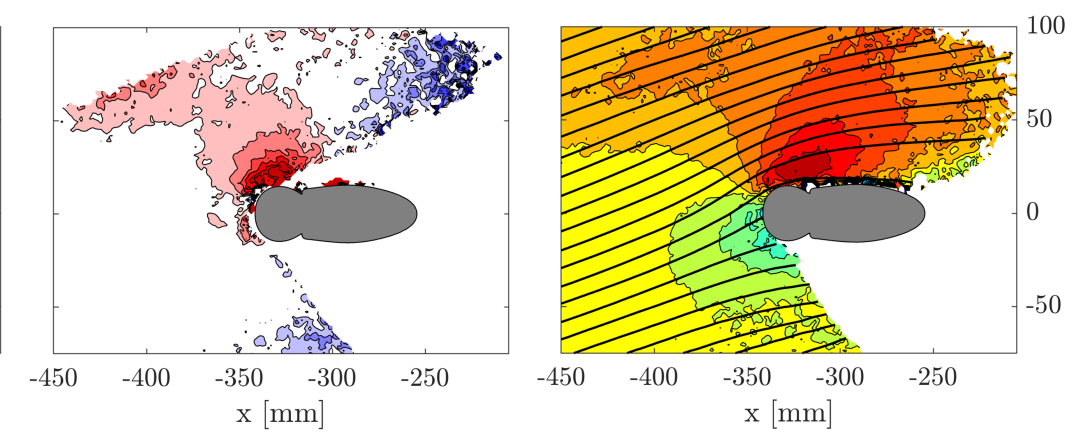

(f) $\beta=18^{\circ}$

Figure 5: (Continued) Time-average velocity contours in $x y$-planes at hub height $(z=342.5 \mathrm{~mm})$, along with 2D surface streamlines. Velocity contours show the normalized axial velocity component in the bicycle wheel reference frame. (Left) Baseline tire. (Right) Option tire. (Center) Delta $=u_{\text {baseline }}-u_{\text {option }}$.

\subsection{Pressure data}

Following the analysis of the velocity data, we briefly investigate the pressure distribution over tire and rim profile. Similar to the previous assessment, the analysis starts in a horizontal $x y$-plane at hub height $(z=342.5 \mathrm{~mm})$. Because both tire options behave similar for moderate yaw angles, and data close to the surface is lacking for high yaw angles on the baseline tire, we limit ourselves to the study of the (slick) option tire here.

Figure 6 shows the pressure profiles on the leeward side of the wheel for $\beta=[0$, $8,16]^{\circ}$. The pressure profiles are characterized by a steep pressure reduction over the upstream tire section. As the yaw angle increases, the pressure reduction gains in magnitude, signifying greater suction with increasing yaw angle. The location of minimum $C_{P}$ moves downstream as the yaw angle grows. At $0^{\circ}$ yaw the minimum pressure of $C_{P}=-0.7$ is recorded at $x / c=0.06$, before approaching an approximately constant value of $C_{P}=-0.5$ further downstream. At $8^{\circ}$ yaw instead, the minimum pressure coefficient exceeds a value of -1 , and it is obtained slightly further downstream $(x / c=0.13)$. Increasing the yaw angle further to $16^{\circ}$, the pressure coefficient drops to a minimum of $C_{P}=-1.75$ at

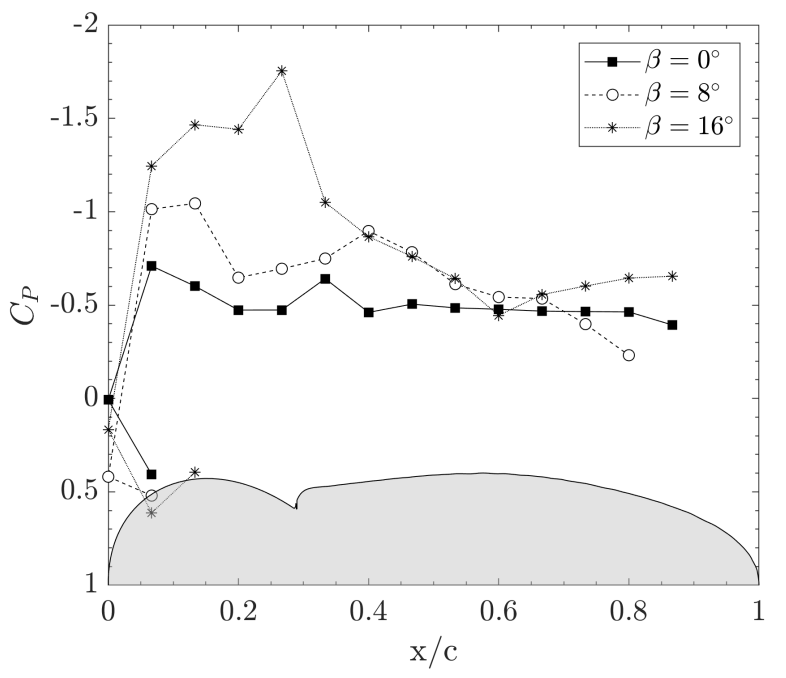

Figure 6: $C_{P}$ profiles over the tire-rim surface at hubheight $(z=342.5 \mathrm{~mm})$ for the option tire (GP TT). 
$x / c=0.26$, followed by an adverse pressure gradient approaching $C_{P}=-0.5$ at $x / c=0.6$.

Complementing the above pressure profiles in the wheel center plane, $3 \mathrm{D}$ iso-surfaces of pressure coefficient are shown for the three selected cases in Figure Z, along with axial velocity contours on the upper and lower boundary of the measurement volume. For each case, two iso-surfaces are plotted: the first, at $C_{P}$ $=0.35$ (red) indicates the volume of increased pressure resulting from the flow deceleration upstream of the wheel. The second, at $C_{P}=-0.6$ (blue) is selected to visualize the volume of reduced pressure induced by the flow acceleration alongside the wheel. Comparing the latter iso-surface for the three yaw angles confirms the growth of the low-pressure region with increasing yaw angle, signaling a likewise increase in side force.
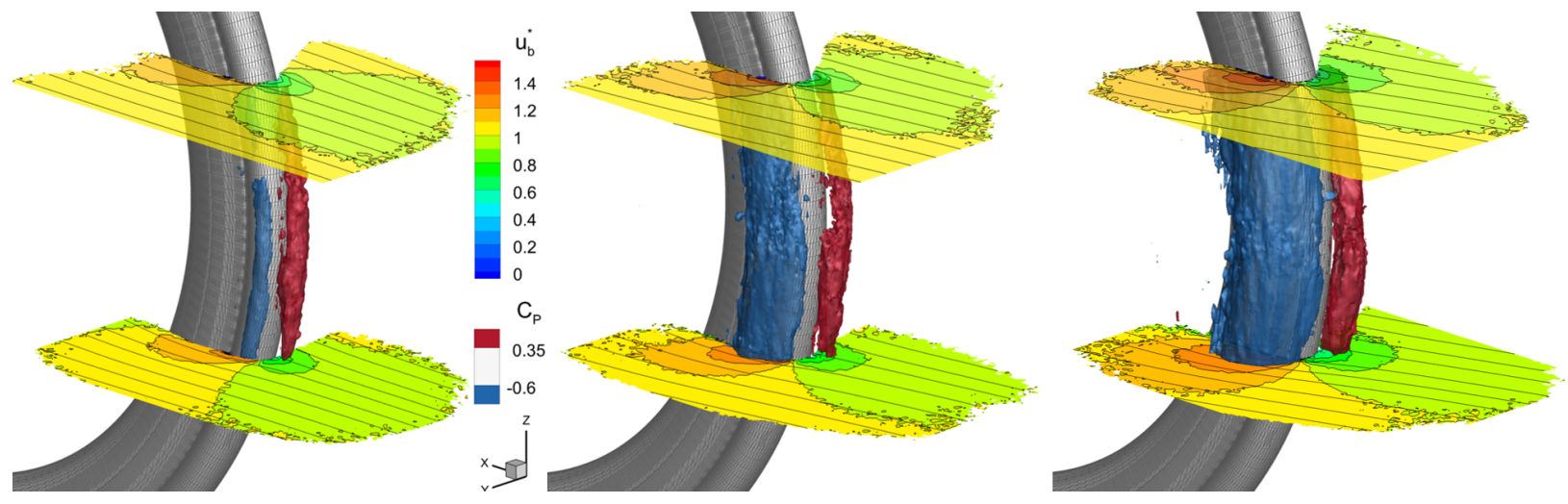

Figure 7: Axial velocity contours in horizontal planes at $z=[242.5,442.5] \mathrm{mm}$ along with 3D pressure iso-surfaces of $C_{P}=0.35$ (red) and -0.6 (blue). Data shown for wheel fitted with option tire at $\beta=0^{\circ}$ (left), $8^{\circ}$ (center) and $16^{\circ}$ (right).

The observed trend is qualitatively in line with the force data presented in Section 4.1$]$ which indicated a monotonically increasing side force magnitude with increasing yaw angle in this range. It further supports the theory that the tire-rim profile in crosswind indeed behaves like an airfoil at incidence, thereby making the wheel work like a sail.

\section{Conclusions}

The aerodynamic characteristics of a state-of-the-art road cycling wheel in cross wind have been assessed experimentally by means of force measurements and a full-scale $3 \mathrm{D}$ velocimetry investigation. Additionally, the interaction of rim and tire is studied by comparison of a textured and a slick tire. The resistive force on the isolated wheel is highest at moderate yaw angles of $\beta=2^{\circ} \pm 4^{\circ}$. Outside this range, the aerodynamic resistance of the wheel is reduced as the yaw angle magnitude increases. Analysis of the velocimetry data confirms that the reduction in resistance measured by a force balance is indeed a consequence of the "saileffect", resulting from a substantial side-force on the wheel at yaw incidence. The benefit of this effect is limited by the stall angle of the tire-rim combination, which is found to be dependent on the tire choice. The two tested tires behave similarly for negative yaw angles, with the flow separating around $\beta=-14^{\circ}$. For positive yaw angles, the slick tire performs significantly better, increasing the separation angle from $14^{\circ}$ to $20^{\circ}$ with respect to the profiled baseline tire.

\section{Acknowledgements}

We thank Thomas Koep on behalf of DT Swiss for providing the wheels for the study. Likewise, we thank Dennis Bruikman, Peter Duyndam and Frits Donker Duyvis for the invaluable technical support. This research is supported by LaVision $\mathrm{GmbH}$. 


\section{References}

Agüera N, Cafiero G, Astarita T, and Discetti S (2016) Ensemble 3D PTV for high resolution turbulent statistics. Measurement Science and Technology 27:124011

Alons HJ (2008) OJF External Balance. Report NLR-CR-2008-695. Nederlands Lucht- en Ruimtevaartcentrum (National Aerospace Laboratory, NLR)

Barry N (2018) A New Method for Analysing the Effect of Environmental Wind on Real World Aerodynamic Performance in Cycling. in 12th Conference of the International Sports Engineering Association. volume 2. page 211

Barry N, Burton D, Crouch T, Sheridan J, and Luescher R (2012) Effect of crosswinds and wheel selection on the aerodynamic behavior of a cyclist. in Engineering of Sport Conference 2012. volume 34. pages $20-25$

Brownlie L, Ostafichuk P, Tews E, Muller H, Briggs E, and Franks K (2010) The wind-averaged aerodynamic drag of competitive time trial cycling helmets. Procedia Engineering 2:2419-2424

Cant G (2014) Back to the wind tunnel. www.swissside.com/blogs/news/back-to-the-wind-tunnel Accessed on 17.05.2021

Cooper KR (2003) Truck aerodynamics reborn-lessons from the past. SAE transactions 112:132-142

Crane R and Morton C (2018) Drag and Side Force Analysis on Bicycle Wheel-Tire Combinations. Journal of Fluids Engineering-Transactions of the Asme 140

Giaquinta D (2018) The Flow Topology of the Ahmed Body in Cross-Wind: An Experimental Investigation by means of Robotic Volumetric PIV. MSc Thesis, TU Delft

Godo M, Corson D, and Legensky S (2010) A comparative aerodynamic study of commercial bicycle wheels using CFD. in 48th AIAA Aerospace Sciences Meeting Including the New Horizons Forum and Aerospace Exposition. page 1431

Greenwell DI, Wood NJ, Bridge EKL, and Addy RJ (1995) Aerodynamic Characteristics of Low-Drag Bicycle Wheels. Aeronautical Journal 99:109-120

Jux C, Sciacchitano A, and Scarano F (2020) Flow pressure evaluation on generic surfaces by robotic volumetric PTV. Measurement Science and Technology

Kyle CR and Burke E (1984) Improving the Racing Bicycle. Mechanical Engineering 106:34-45

Lukes RA, Chin SB, and Haake SJ (2005) The understanding and development of cycling aerodynamics. Sports Engineering 8:59-74

Malizia F and Blocken B (2020a) Bicycle aerodynamics: History, state-of-the-art and future perspectives. Journal of Wind Engineering and Industrial Aerodynamics 200:104134

Malizia F and Blocken B (2020b) CFD simulations of an isolated cycling spoked wheel: The impact of wheel/ground contact modeling in crosswind conditions. European Journal of Mechanics B-Fluids $84: 487-495$

Malizia F, Montazeri H, and Blocken B (2019) CFD simulations of spoked wheel aerodynamics in cycling: Impact of computational parameters. Journal of Wind Engineering and Industrial Aerodynamics 194

Scarano F, Ghaemi S, Caridi GCA, Bosbach J, Dierksheide U, and Sciacchitano A (2015) On the use of helium-filled soap bubbles for large-scale tomographic PIV in wind tunnel experiments. Experiments in Fluids 56:42

Schanz D, Gesemann S, and Schröder A (2016) Shake-The-Box: Lagrangian particle tracking at high particle image densities. Experiments in Fluids 57:70 
Schneiders JFG, Scarano F, Jux C, and Sciacchitano A (2018) Coaxial volumetric velocimetry. Measurement Science and Technology 29:065201

Sciacchitano A and Scarano F (2014) Elimination of PIV light reflections via a temporal high pass filter. Measurement Science and Technology 25:084009

Tew GS and Sayers AT (1999) Aerodynamics of yawed racing cycle wheels. Journal of Wind Engineering and Industrial Aerodynamics 82:209-222

Zdravkovich M (1992) Aerodynamics of bicycle wheel and frame. Journal of Wind Engineering and Industrial Aerodynamics 40:55-70 